\title{
NIVEL DE CONOCIMIENTO Y ACTITUDES DE LOS PACIENTES RESPECTO DEL CONSENTIMIENTO INFORMADO PARA LA DOCENCIA EN EL DEPARTAMENTO DE MEDICINA INTERNA DE UN HOSPITAL GENERAL DEL MINISTERIO DE SALUD
}

\author{
LEVEL OF KNOWLEDGE AND ATTITUDES OF PATIENTS \\ REGARD OF THE INFORMED CONSENT FOR TEACHING IN \\ THE DEPARTMENT OF INTERNAL MEDICINE OF A GENERAL \\ HOSPITAL OF THE MINISTRY OF HEALTH
}

\author{
MARIO CARRIÓN CHAMBILLA \\ Hospital Nacional Arzobispo Loayza \\ AGUSTÍN IZA STOLL \\ Universidad Norbert Wiener \\ ALEJANDRA LA TORRE MATUK \\ Hospital Nacional Arzobispo Loayza
}

\section{RESUMEN}

Los hospitales nacionales han servido para propósitos de docencia de la Medicina, y de otras profesiones de la salud, prácticamente desde que iniciaron su funcionamiento. En los últimos años se han formulado diversos dispositivos legales y administrativos que norman los derechos de los pacientes en relación a la docencia; ellos establecen que debe constar el consentimiento escrito del paciente en la historia clínica. Se formuló una encuesta a 239 pacientes adultos internados en un hospital docente, y en el 99,5\% no se encontró el consentimiento informado en la historia clínica; el 100 no conocía sus derechos, pero sabía que en el hospital se hacía docencia; el 98,6 \% manifestó que recibieron un trato respetuoso de los alumnos de las ciencias de la salud, y todos estaban dispuestos a colaborar en el entrenamiento de los estudiantes. Se hace necesario que las autoridades hospitalarias y de las universidades supervisen que los profesionales de la salud docentes informen adecuadamente a los pacientes sobre estos derechos y obtengan su consentimiento.

Palabras clave: hospital, pacientes, educación médica, consentimiento informado, recursos humanos en salud.

\section{ABSTRACT}

The national hospitals have served as teaching purpose of the medicine and of the other professions of the health since the beginning of their operations. In the last years several legal and administrative mechanisms of the right of patients regarding to the teaching have being developed. These mechanisms establish that is necessary the written consent of the patient in the medical record. A poll was formulated to 
239 adult patients hospitalized in a hospital; the $99,5 \%$ of them had not the informed consent in the clinic history; the $100 \%$ of them did not know their rights, but they knew that in the hospital the learning was done; the 98,6 \% indicated that they received a respectful treatment from resource in health.

Key words: hospital, patients, medical educations, informed consent, human resource in health.

\section{INTRODUCCIÓN}

La atención de los pacientes debe darse dentro de un marco de respeto a los derechos humanos. La Declaración Universal sobre Bioética y Derechos Humanos de la UnEsCo, en su artículo 6, establece que «toda intervención médica preventiva, diagnóstica y terapéutica sólo habrá de llevarse a cabo previo consentimiento libre e informado de la persona interesada, basado en la información adecuada. Cuando proceda, el consentimiento debería ser expreso y la persona interesada podrá revocarlo en todo momento y por cualquier motivo, sin que esto entrańe para ella desventaja o perjuicio alguno» ${ }^{1}$.

Si bien no se hace una referencia explícita a las actividades de docencia como se hace con las de investigación, es cierto también que las actividades de docencia podrían tener impacto en el resultado de la atención de los pacientes, por lo que el paciente debe conocer la implicancia de la participación del estudiante y tomar una decisión sobre la participación de este durante la atención.

El consentimiento informado es un proceso por el cual una persona acepta participar en alguna actividad médica (asistencial, de investigación o docencia) "conociendo los riesgos, beneficios, consecuencias o problemas que se puedan presentar durante el desarrollo de la misma ${ }^{2}$. Según Resolución 078-2016/MinsA, se aprueba el formato del Consentimiento Informado para las actividades de docencia durante la atención de salud, el cual deberá incorporarse a la historia clínica, además del instructivo para su adecuado llenado ${ }^{3}$.

El Reglamento de Establecimientos de Salud y Servicios Médicos de Apoyo, aprobado mediante D. S. N N $^{\circ}$ 13-2006-SA, Minsa, en el artículo $114 .^{\circ}$, referido a la participación de las personas en actividades de docencia, refiere que «en el momento del ingreso del paciente a un establecimiento de salud o servicio médico de apoyo, en los cuales se realizan actividades de docencia, se deberá preguntar al paciente si desea aceptar o rehusarse a participar en estas actividades. En la historia clínica deberá constar el consentimiento escrito del paciente, o de la persona llamada legalmente a darlo, de participar en las actividades de docencia» ${ }^{4}$.

La Ley 29414 (2009), relativa a los derechos de las personas usuarias de los servicios de salud, establece en su artículo $15 .^{\circ}$ que toda persona tiene derecho a "otorgar su consentimiento informado, libre y voluntario, sin que medie ningún mecanismo que vicie su voluntad, para el procedimiento o tratamiento de salud, en especial cuando se trate de exploración, tratamiento o exhibición con fines docentes». La citada ley además establece que «el consentimiento informado debe constar 
por escrito en un documento oficial que visibilice el proceso de información y decisión. Si la persona no supiere firmar, imprimirá su huella digital ${ }^{5}$.

En agosto de 2015 se publicó el Reglamento de la Ley 29414, que en su artículo 19 señala que antes de la exploración, tratamiento o exhibición de imágenes con fines docentes se debe obtener la firma del consentimiento informado y que el médico tratante debe «garantizar el respeto a la privacidad y pudor del paciente». Además, en su artículo 24 menciona que «el consentimiento escrito deberá ejecutarse de forma obligatoria cuando se trate de exploración, tratamiento o exhibición de imágenes con fines docentes" 6 .

El D. S. 031-2014-SA, que aprueba el Reglamento de Infracciones y Sanciones de la Superintendencia Nacional de Salud-Susalud, en el anexo III respecto de infracciones aplicables a las IPRESs, considera una infracción el «realizar la exploración, tratamiento o exhibición con fines docentes $\sin$ consentimiento informado por escrito», la cual puede ser sancionada con una multa de hasta 100 UIT $^{7}$.

En 2011, las autoridades del hospital en el que se desarrolló el presente estudio, mediante resolución directoral, aprobaron el formato de consentimiento informado para la docencia, el cual, luego de su implementación, no ha logrado ser incorporado como un procedimiento habitual en la práctica diaria de la institución.

El consentimiento informado es un documento que es utilizado como un instrumento para obtener el permiso para desarrollar una determinada acción sobre una persona. Desde hace varios ańos existe el consentimiento informado para procedimientos invasivos o no invasivos; el cual se incorporó el consentimiento informado para participar como sujetos de investigación. En los últimos años se está considerando el consentimiento informado para las actividades de docencia, sin embargo, en nuestro medio no está muy difundido su uso durante las actividades de docencia en servicio.

Por otra parte, en la práctica diaria de la institución es frecuente que los pacientes sean sujeto de docencia por muchos estudiantes de las diversas carreras de las ciencias de la salud, pero en la mayoría de ellos no se ha solicitado el consentimiento informado por escrito según dicta la norma. Existe la percepción entre los profesionales de la salud de que los pacientes no estarían dispuestos a participar de las actividades de docencia con los estudiantes. Por ello estimamos necesario conocer la opinión, así como el nivel de conocimientos de los pacientes, sujetos de docencia, respecto de este tema.

El objetivo del presente estudio es determinar el nivel de conocimientos y actitudes de los pacientes respecto del consentimiento informado para la docencia en un hospital general del Minsa.

\section{MÉTODOS}

El presente estudio fue de tipo observacional, prospectivo, transversal, descriptivo. La población estuvo conformada por los pacientes hospitalizados en un establecimiento de Salud Nivel III-1 del Minsa. La muestra a la cual se aplicó la encuesta estuvo conformada por 239 pacientes hospitalizados en los servicios del Departamento de Medicina Interna.

Previamente se obtuvo las autorizaciones respectivas de las áreas pertinentes de 
la institución hospitalaria, así como del paciente. Se aplicó una encuesta anónima estructurada con preguntas de respuesta cerrada, y en algunos casos con alternativas múltiples. La encuesta se aplicó durante los meses de agosto y setiembre de 2016; se consideró a un solo paciente por cama de hospitalización, de tal manera que no se entrevistó a más de un paciente por cama durante el periodo de la aplicación del instrumento.

Una encuestadora, estudiante de las ciencias de la salud previamente capacitada por el equipo de investigación en el instrumento a aplicar, se entrevistó con cada uno de los participantes. Les explicó en qué consistía el estudio y solicitó su autorización para participar en él a través de la firma de un consenti- miento informado. Se procedió a leer las preguntas del cuestionario y se resolvieron las dudas que podrían surgir respecto de la comprensión del mismo. La encuestadora completó el formulario de la encuesta en base a las respuestas de los participantes. Se consideraron para la presente investigación aquellos pacientes que estuvieran hospitalizados por lo menos 72 horas.

Luego de obtenidos los registros a través de la encuesta, estos fueron incorporados a una base de datos en Excel, la misma que fue trasladada al programa SPSS versión 22 . Se realizó el análisis descriptivo presentando las variables cualitativas con frecuencias y las variables cuantitativas con sus respectivas medidas de tendencia central y de dispersión.

TABLA

Resultados de la encuesta a 239 pacientes hospitalizados

\begin{tabular}{lcc}
\hline \multirow{2}{*}{ Pregunta } & NO & Sí \\
& $(\%)$ & $(\%)$
\end{tabular}

¿Conoce sus derechos, como usuario del hospital, en relación a las actividades de docencia de los estudiantes de las ciencias de la salud?

$99,6 \quad 0,4$

¿Sabe usted si existe alguna ley que regule y proteja los derechos de las personas durante las actividades de docencia, en un establecimiento de salud (hospital)?

¿Se le ha mencionado o entregado alguna información donde se mencione sus derechos en relación a las actividades de docencia con los estudiantes de las ciencias de la salud?

¿Sabe usted que este es un hospital dedicado a la docencia de estudiantes (pregrado o posgrado) de las ciencias de la salud?

¿Sabe usted que durante su hospitalización podría ser atendido por estudiantes de las ciencias de la salud?

¿El trato que ha recibido de los estudiantes ha sido respetuoso o muy respetuoso?
$2.1 \quad 97,9$

$5,4 \quad 94,6$

100

$1,7 \quad 98,3$

$100 \quad 0$




\section{RESULTADOS}

Las encuestas fueron aplicadas entre agosto y setiembre de 2016, previo consentimiento verbal en un $100 \%$, y por escrito de 161 participantes $(67,4 \%)$; esto, debido a que tenían canalizada una vía venosa periférica en el miembro superior dominante o incapacidad funcional para escribir.

En el presente estudio se entrevistaron a 239 pacientes pertenecientes a los 9 servicios de hospitalización del Departamento de Medicina Interna. El 50,6 \% fueron del sexo femenino y el rango de edad fue de 17 a 102 años con una media de 56,95 años, mediana $=8$ años y $\mathrm{DE}=18.56$. El $74,1 \%$ reportaron tener secundaria completa o incompleta, seguido de un $18,4 \%$ para primaria completa o incompleta. Al consultar sobre las veces que habían estado hospitalizados previamente en esta institución, 136 (56,9\%) reportaron que era la primera vez. En el 99,5\% de las historias clínicas no se encontró firmado el consentimiento informado para actividades de docencia.

Como se aprecia en la Tabla, todos los pacientes encuestados desconocían los dispositivos legales que protegen sus derechos como pacientes en relación con la docencia, y tampoco habían recibido información con referencia al tema. Sin embargo, un alto porcentaje $(97,9 \%)$ admitió conocer que se realiza docencia en el hospital y que puede ser atendido por alumnos durante su hospitalización, y el $98,3 \%$ manifestó haber recibido un trato respetuoso por parte de los estudiantes. Además, el 98,7 \% mencionó que no sabía que podría negarse a ser entrevistado o evaluado por los estudiantes.

$\mathrm{Al}$ ser consultados respecto a si la condición de hospital docente podría favo- recer el cuidado de su salud, el $100 \%$ contestó que era favorecedor o muy favorecedor. Así mismo, el 100 \% mencionó estar dispuesto o muy dispuesto a colaborar en la formación de los estudiantes si se lo solicitasen, y el 79,5\%, que estaría dispuesto a relatar su historia clínica a los estudiantes de las ciencias de la salud más de una vez. Por otra parte, el 99,6\% considera importante o muy importante el hecho de que los estudiantes puedan conversar sobre su historia clínica o desarrollar algún procedimiento con supervisión para la formación de los futuros profesionales.

El 94,1\% de los encuestados manifestó que durante esta hospitalización nunca le habían solicitado su autorización para que colabore con los estudiantes de las ciencias de la salud, y que este pedido fue solo en forma verbal en el 5,9\%. En contraposición, el 99,2 \% de los participantes considera que sí es necesario solicitar su autorización para que los estudiantes de las ciencias de la salud desarrollen actividades de docencia.

\section{DISCUSIÓN}

El consentimiento informado es el instrumento mediante el cual se protege el derecho del paciente a participar en la adopción de decisiones sobre su salud y se inspira en la relación médico-paciente. Su obtención debería ser una práctica común y familiar tanto para el personal de salud como para los pacientes.

La importancia de la aplicación del consentimiento informado para las actividades de docencia se sustenta en la expresión del respeto de los profesionales de las ciencias de la salud respecto al derecho de los pacientes de ser sujetos de docencia, fortaleciendo con ello la 
dignidad del ser humano. Además, al ser un requisito que establece el marco normativo de los establecimientos de salud para que los pacientes puedan participar en actividades de docencia, permitirá prevenir las evidentes repercusiones legales que podrían resultar de su falta de aplicación $n^{7,8}$.

Así mismo, en 2011 se aprobó el formato de consentimiento informado para las actividades de docencia, el cual sin embargo ha tenido una baja tasa de aplicación. En ese sentido, conocer el nivel de conocimientos y actitudes respecto del consentimiento informado para la docencia podría ayudar a identificar algunos aspectos que al ser abordados permitirían su mayor aplicación en la práctica diaria.

La responsabilidad de llevar a cabo el proceso de consulta para el consentimiento informado es del profesional de la salud docente, quien debe garantizar el derecho de información y el derecho de la libertad de decisión de la persona usuaria de los servicios de salud. De hecho, según el mismo documento, Consentimiento Informado para las actividades de docencia durante la atención de salud del MINSA ${ }^{3}$, el formato debe ser entregado y explicado por el profesional de las ciencias de la salud, que es también docente.

La obtención del consentimiento informado es una expresión del respeto de la autonomía de las personas de poder decidir sobre aquello que es de su competencia; en el caso particular de la docencia se convierte en una herramienta importante en la formación de los futuros profesionales, pues permite la adquisición de competencias de comunicación y de consolidación del valor de respeto por los seres humanos, quienes pueden ser sujetos, pero no objetos de docencia.
En el presente estudio, casi la totalidad de los pacientes encuestados conoce que el hospital donde se desarrolló el estudio es una institución dedicada a la docencia, y, por ello, podrían ser atendidos por estudiantes de las ciencias de la salud.

La totalidad de las personas encuestadas manifestaron que estarían dispuestas a colaborar con los estudiantes y las actividades de docencia, siempre que se les solicite su autorización. Este importante grado de aceptabilidad a participar en las actividades de docencia se podría también explicar, entre otros aspectos, en que el 98,3\% de los participantes mencionan que el trato recibido de los estudiantes fue respetuoso?.

Pero también resulta pertinente considerar cuál es el comportamiento o la interpretación de los médicos con respecto a su rol en el consentimiento informado. Zavala encontró, entre 1486 médicos que se presentaron al examen de residentado en el año 2010, que la mayoría (84\%) respondió que respetar la autonomía del paciente significa mejorar la comunicación médico paciente; igualmente, halló que tres de cada cuatro indicaron que obtener el consentimiento informado es un imperativo ético. No obstante, dentro de la heterogeneidad de esta población en edad, universidad de origen y año de egreso, se identificaron deficiencias en el conocimiento del consentimiento informado ${ }^{10}$.

Es indispensable remarcar que la práctica de la Medicina requiere que se realice respetando la dignidad de los pacientes, su autonomía y su libre determinación. Solicitar a los pacientes su autorización para ser partícipe de la enseñanza de los alumnos de Medicina no es una práctica común en nuestros hospitales, y debería imple- 
mentarse apoyada en documentos legales nacionales y de organismos internacionales, como la Asociación Médica Mundial y las Naciones Unidas, que inciden en el respeto a los derechos humanos ${ }^{11}$.

La totalidad de los participantes del estudio considera que la práctica de la docencia en una institución de salud podría favorecer el cuidado de su salud. Esta disposición se debe aprovechar para mejorar la relación con los pacientes, brindarles una adecuada atención y a la vez darles a conocer sus derechos. Además, podría ser útil para lograr una mayor adherencia al uso del consentimiento informado de parte de los profesionales de las ciencias de la salud, los que suelen pensar que los pacientes no estarían de acuerdo en participar en actividades de formación de los estudiantes.

Dado que el $100 \%$ de los pacientes ignoran que existe una ley que regula y protege sus derechos respecto de las actividades de docencia, sería necesario que la institución hospitalaria implemente alguna estrategia que garantice a los pacientes el acceso a esta información, de tal forma que puedan ejercer su derecho a tomar una decisión informada respecto de su participación en las actividades de docencia.

El $100 \%$, incluyendo a los que habían estado hospitalizados previamente, y con cualquier nivel de educación, consideró importante o muy importante el desarrollo de las actividades de docencia en la formación de futuros profesionales, hecho que debe ser tomado como un factor que favorece la implementación de las actividades de docencia con los pacientes, pero que al mismo tiempo debe ser tomada con mucha responsabilidad en la medida que los pacientes la consideran como de gran importancia en la formación de los estudiantes.

Los hallazgos de este estudio muestran resultados favorables respecto de las actitudes que tienen los pacientes hacia las actividades de docencia de los estudiantes de las ciencias de la salud. Creemos que lo hallado permitirá implementar la aplicación del consentimiento informado para la docencia con un menor grado de resistencia de parte de los profesionales de la salud.

Sin embargo, se debe resaltar que este estudio abordó una población en particular, la que se atiende en un servicio de Medicina Interna. Será importante aplicar el mismo estudio en otros escenarios que podrían tener una respuesta diferente, de acuerdo con las características de la población que es atendida, por ejemplo en pediatría, ginecología o urología. Así mismo, podríamos analizar el nivel de participación en otras circunstancias de la atención, como en consulta externa, en emergencia o en las unidades de cuidados intensivos.

\section{CONCLUSIONES}

Casi la totalidad de los pacientes encuestados identifica al establecimiento de salud como una institución dedicada a la docencia y así mismo que estarían dispuestos a colaborar con las actividades de docencia.

La totalidad de pacientes ignora que existe una ley que regula y protege sus derechos referidos a las actividades de docencia; refieren además que la institución tampoco les ha informado sobre los mismos. 
Casi todos los pacientes consideran importante las actividades de docencia para la formación de los futuros profesionales, así como que la condición de ser atendidos en un hospital docente podría favorecer el cuidado de su salud.

Se hace necesario que las autoridades del hospital y las universitarias entrenen y supervisen que los docentes, que también son trabajadores del hospital, cumplan con estas disposiciones que respetan los derechos de los pacientes.

\section{REFERENCIAS BIBLIOGRÁFICAS}

1. UneSCO. Declaración universal sobre Bioética y Derechos Humanos. Resolución aprobada, previo informe de la Comisión III, en la 18. a Sesión Plenaria del 19 de octubre de 2005. Disponible en: <http://portal.unesco. org/es/ev.php>.

2. Mondragon-Barrios L. Consentimiento informado: una praxis dialógica para la investigación. Rev Invest Clin 2009; 61(1):73-82.

3. Ministerio de Salud. Consentimiento Informado para las actividades de docencia durante la atención de salud. Resolución del Ministerio de Salud 078-2016/Minsa. Disponible en: $<$ www.minsa.gob.pe>.

4. Ministerio de Salud. Reglamento de Establecimientos de Salud y Servicios Médicos de Apoyo, aprobado mediante Decreto Supremo N. ${ }^{\circ} 013-2006-S A$. Disponible en: <www.minsa.gob.pe>.

5. Ministerio de Salud. Ley 29414, ley que establece los derechos de las personas usuarias de los servicios de salud. Disponible en: <www.minsa.gob.pe>.
6. Ministerio de Salud. Reglamento de la Ley 29414, ley que establece los derechos de las personas usuarias de los servicios de salud. Consulta: 13 agosto 2015. Disponible en <www.minsa. gob.pe>.

7. Ministerio de Salud. Decreto Supremo 031-2014-SA, que aprueba el Reglamento de Infracciones y Sanciones de la Superintendencia Nacional de Salud-susalud. Disponible en: <www. minsa.gob.pe >.

8. Carrasco-Aldunate P, Rubio-Acuña M, Fuentes-Olavarría D. Consentimiento informado: un pilar de la investigación clínica. Aquichan. 12(1):32-41;2012 DOI: <https://doi. org/10.5294/32-41>.

9. Zárate E. Los derechos de los pacientes y el Consentimiento Informado en Perú. SITUA, Revista Semestral de la Facultad de Medicina Humana-UNSAAC. 23; 1-10; 2004.

10. Zavala S, Sogi C, Cárdenas M, Delgado A. Autonomía del paciente y derecho a rehusar tratamientos: Conocimiento en Médicos Jóvenes Peruanos (encuesta 2010). Acta Med Per 29(2):7077; 2012.

11. Gianella G. Los derechos humanos y el consentimiento informado en la práctica clínica: más allá del derecho a la salud. Rev Peru Med Exp Salud Pública. 2013; 30(2):315-19. 